\title{
Usefulness of preoperative FDG-PET for detection of gastric cancer
}

\author{
Kota Mukai ${ }^{1}$, Yasuhiko Ishida $^{1}$, Kunio Okajima $^{1}$, Hiroshi Isozaki $^{2}$, Tsukasa Morimoto ${ }^{3}$, and Shoj Nishiyama ${ }^{3}$ \\ ${ }^{1}$ Department of Surgery, Himeji Central Hospital, 2-36 Miyake, Shikama-ku, Himeji 672-8501, Japan \\ ${ }^{2}$ Department of Surgery, Oomoto Hospital, Okayama, Japan \\ ${ }^{3}$ Department of Radiology, Himeji Central Hospital, Himeji, Japan
}

\begin{abstract}
Background. Positron emission tomography (PET), using 18F-fluoro-2-deoxy-D-glucose (FDG) as a tracer, can detect malignant neoplasms with altered glucose metabolism. To clarify the usefulness of FDG-PET for detecting gastric cancer, we evaluated preoperative PET imaging in gastric cancer patients.

Methods. Sixty-two gastric cancer patients who underwent FDG-PET imaging and gastric resection with lymphadenectomy were evaluated.

Results. For primary tumor assessment by PET, detection rates were significantly different in the following order: tumor size $30 \mathrm{~mm}$ or more $(76.7 \%)>$ tumor size less than $30 \mathrm{~mm}$ (16.8\%); advanced gastric cancer (AGC, 82.9\%) > early gastric cancer (EGC; $25.9 \%$ ); with nodal involvement $(\mathbf{7 9 . 3} \%$ ) > without nodal involvement $(39.4 \%)$. In EGCs, the detection rate of the intestinal type, according to Lauren's classification $(43.8 \%)$ was significantly higher than that of the diffuse type $(0 \%)$. Two of the 7 EGC patients who were PET-positive had nodal involvement and their tumors were the intestinal type. For the assessment of nodal involvement, the accuracy of nodal involvement detection was $67.7 \%$ with PET and $75.8 \%$ with computed tomography (CT). Preoperative FDG-PET revealed colon cancer in 2 patients, adrenal tumor in 1 patient, lung cancer in 1 patient, and lung metastasis in 1 patient.

Conclusion. Larger or more advanced tumors with nodal involvement had a higher detection rate by PET. In EGCs, only the intestinal type was detectable by PET. PET-positive EGC may be aggressive, and an adequate lymphadenectomy must be done. Preoperative PET was useful for the detection of other malignancies and distant metastasis.
\end{abstract}

Key words FDG-PET · Gastric cancer · Preoperative diagnosis $\cdot$ Nodal involvement $\cdot$ Distant metastasis

Offprint requests to: $\mathrm{K}$. Mukai

Received: October 17, 2005 / Accepted: March 20, 2006

\section{Introduction}

Positron emission tomography (PET), using 18F-fluoro2-deoxy-D-glucose (FDG) as a tracer, can detect malignant neoplasms with altered glucose metabolism. In recent years, the fusion of images from FDG-PET with those of computed tomography (CT) has been reported to be useful for the detection of the sites of such malignancies.

There are a few reports [1-4] on the usefulness of PET for the diagnosis of gastric cancer. The rate of detection of gastric cancer by PET is reported to be lower than that for other malignancies. The physiological accumulation of FDG in the stomach may prevent the detection of small malignancies [5]. Thus, the usefulness of PET for detecting gastric cancer has remained uncertain. In this study, we evaluated the clinical usefulness of preoperative FDG-PET for gastric cancer patients.

\section{Patients and methods}

A total of 62 consecutive gastric cancer patients who underwent gastric resection with lymphadenectomy at Himeji Central Hospital, from July 2004 to June 2005 , were enrolled in this study. They consisted of 42 men and 20 women (average age, 67.6 years). FDGPET was performed preoperatively along with other examinations.

\section{FDG-PET imaging}

PET images were obtained using a PET scanner (Allegro-Philips; Eindhoven, The Netherlands) with a $57.6-\mathrm{cm}$ transaxial field of view and an $18.0-\mathrm{cm}$ axial field of view. All patients fasted for more than $6 \mathrm{~h}$ before their scan, and a blood sample was taken to confirm normoglycemia (less than $200 \mathrm{mg} / \mathrm{dl}$ ). Sixty min before 
the PET scanning, $4.44 \mathrm{Mbq} / \mathrm{kg}$ of FDG was injected. Emission time was $2.5 \mathrm{~min} /$ one bed and transmission time was $23 \mathrm{~s} /$ one bed. Subsequently, a fusion of the FDG-PET image combined with the CT image was obtained. A nuclear medicine radiologist, who was not aware of the patient data, evaluated each image by visual analysis. Another radiologist confirmed that the diagnosis made by the first radiologist was reproducible.

Total gastrectomy was performed in 22 patients, distal gastrectomy in 31 patients, proximal gastrectomy in 4 patients, and other partial gastrectomies in 3 patients. D2 lymphadenectomy, according to the guidelines of the Japanese Gastric Cancer Association [6], was performed in 40 patients, $\mathrm{D} 1+\alpha, \beta$ in 16 patients, and $\mathrm{D} 0$ in 6 patients. Curative resection was performed in 53 patients and noncurative resection in 9 patients.

Clinicopathological factors were evaluated in accordance with the Japanese classification of gastric carcinoma [6]. The histological type of gastric cancer was divided into two types, according to Lauren's classification [7]. The intestinal type consisted of papillary adenocarcinoma (pap), well-differentiated tubular adenocarcinoma (tub1), and moderately differentiated tubular adenocarcinoma (tub2). The diffuse type consisted of poorly differentiated adenocarcinoma (por), signet-ring cell carcinoma (sig), and mucinous adenocarcinoma (muc).

Statistical analysis was performed by the $\chi^{2}$ test, and $P<0.05$ was considered significant. Sensitivity was defined as the number of true-positive cases divided by the sum of the true-positive cases plus the false-negative cases. Specificity was defined as the number of truenegative cases divided by the sum of the true-negative cases plus the false-positive cases.

This study was done according to the revised version of the Declaration of Helsinki (2000).

\section{Results}

Primary tumor assessment by FDG-PET

The detection rate of primary tumor in the stomach according to the FDG-PET results is shown in Table 1. The detection rate of tumors less than $30 \mathrm{~mm}(16.8 \%)$ was significantly lower than that of tumors $30 \mathrm{~mm}$ or over $(76.7 \%)$. The detection rate of early gastric cancer (EGC; $25.9 \%$ ) was significantly lower than that of advanced gastric cancer (AGC; $82.9 \%$ ). The detection rate of gastric cancer with nodal involvement $(79.3 \%)$

Table 1. Detection rate of primary tumor, by FDG-PET, in 62 gastric cancer patients

\begin{tabular}{|c|c|c|c|}
\hline Characteristic & \multicolumn{2}{|c|}{ Percent detected by PET } & $\chi^{2}$ test \\
\hline \multicolumn{4}{|l|}{ Tumor location } \\
\hline Upper & \multicolumn{2}{|l|}{$65.2(15 / 23)$} & \multirow[t]{4}{*}{$P=0.1996$} \\
\hline Middle & $36.4(8 / 22)$ & & \\
\hline Lower & $73.3(11 / 15)$ & & \\
\hline Whole & $50.0(1 / 2)$ & & \\
\hline \multicolumn{4}{|l|}{ Tumor size } \\
\hline$<30 \mathrm{~mm}$ & $16.8(3 / 14)$ & & \multirow[t]{2}{*}{$P<0.0001$} \\
\hline$\geqq 30 \mathrm{~mm}$ & $76.7(33 / 43)$ & & \\
\hline \multicolumn{4}{|c|}{ Depth of invasion } \\
\hline EGC $\mathrm{T} 1$ & \multicolumn{2}{|l|}{$25.9(7 / 27)$} & \multirow[t]{4}{*}{$P<0.0001$} \\
\hline \multirow{3}{*}{$\begin{array}{ll}\text { AGC } & \text { T2 } \\
& \text { T3 }\end{array}$} & $81.3(13 / 16)$ & \multirow{3}{*}{$82.9(29 / 35)$} & \\
\hline & $87.5(14 / 16)$ & & \\
\hline & $66.7(2 / 3)$ & & \\
\hline \multicolumn{4}{|c|}{ Lymph node metastasis } \\
\hline pN0 & $39.4(13 / 33)$ & & \multirow[t]{4}{*}{$P=0.0035$} \\
\hline $\mathrm{pN} 1$ & $71.4(10 / 14)$ & & \\
\hline $\mathrm{pN} 2$ & $84.6(11 / 13)$ & $79.3(23 / 29)$ & \\
\hline $\mathrm{pN} 3$ & $100(2 / 2)$ & & \\
\hline \multicolumn{4}{|l|}{ Histology } \\
\hline Intestinal & \multicolumn{2}{|l|}{$65.5(19 / 29)$} & \\
\hline \multirow{2}{*}{$\begin{array}{l}\text { Tub1 } \\
\text { Tub2 }\end{array}$} & \multirow{2}{*}{\multicolumn{2}{|c|}{$\begin{array}{l}(3 / 9) \\
(16 / 20)\end{array}$}} & \\
\hline & & & \\
\hline Diffuse & \multicolumn{2}{|l|}{$51.5(17 / 33)$} & $P=0.2649$ \\
\hline Por & \multicolumn{2}{|l|}{$(16 / 26)$} & \\
\hline $\mathrm{Sig}$ & \multicolumn{2}{|l|}{$\begin{array}{l}(16 / 26) \\
(0 / 6)\end{array}$} & \\
\hline Muc & \multicolumn{2}{|l|}{$(1 / 1)$} & \\
\hline
\end{tabular}

EGC, early gastric cancer; AGC, advanced gastric cancer; tub 1, well-differentiated adenocarcinoma (ad. ca.); tub 2, moderately differentiated ad. ca.; por, poorly differentiated ad.ca.; sig, signet ring cell carcinoma; muc, mucinous ad.ca. 
Table 2. Detection rate of EGCs (type according to Lauren's classification) by FDG-PET

\begin{tabular}{llcc}
\hline & Intestinal type & Diffuse type & $\chi^{2}$ test \\
\hline EGC & $43.8 \%(7 / 16)$ & $0 \%(0 / 11)$ & $P=0.0216$ \\
AGC & $92.3 \%(12 / 13)$ & $77.3 \%(17 / 22)$ & $P=0.3771$
\end{tabular}

EGC, early gastric cancer; AGC, advanced gastric cancer

Table 3. Relation between primary tumor size and accumulation of FDG in intestinal-type early gastric cancer

\begin{tabular}{|c|c|c|c|}
\hline \multirow{2}{*}{$\frac{\text { Depth }}{\mathrm{M}}$} & \multicolumn{3}{|c|}{ Size of primary tumor $(\mathrm{mm})$} \\
\hline & $\begin{array}{r}\text { 10 } \\
\bigcirc 10 \\
\bigcirc 10\end{array}$ & $\begin{array}{ll}\bigcirc 20 & \bigcirc 27 \\
\bigcirc 20 & \end{array}$ & $\bigcirc 31 \bigcirc 35$ \\
\hline SM & $\bigcirc 10 \bigcirc 16$ & $25 \bigcirc 30$ & $54^{a}$ \\
\hline
\end{tabular}

Black circles, PET (+); white circles, PET (-)

${ }^{a}$ With nodal involvement

was significantly higher than that without nodal involvement $(39.4 \%)$. Overall, the detection rate of the intestinal type (65.5\%) was higher than that of the diffuse type $(51.5 \%)$; however, this difference was not significant. The detection rate of tumors $35 \mathrm{~mm}$ or over of the intestinal type was $100 \%$.

\section{Detection rate of EGCs (type according to Lauren's classification) by FDG-PET (Table 2)}

The detection rate of intestinal-type EGCs (7/16; $43.8 \%$ ) was significantly higher than that of the diffuse type $(0 / 11 ; 0 \%)$. Three EGCs less than $30 \mathrm{~mm}$ in size $(10 \mathrm{~mm}, 20 \mathrm{~mm}$, and $25 \mathrm{~mm})$ were detected by PET (Table 3).

\section{Nodal involvement in EGC patients}

Seven of 27 EGCs were PET-positive. Two of these 7 PET-positive-patients $(28.6 \%)$ had nodal involvement. The primary tumors of these 2 patients were intestinal type. In contrast, 20 of the 27 EGCs were PET-negative. None of these 20 PET-negative-patients had nodal involvement.

\section{Detection of nodal involvement by PET and CT} (Table 4)

The sensitivity of detection of nodal involvement by PET $(34.5 \%)$ was lower than that of CT $(62.1 \%)$. However, the specificity of detection of nodal involvement by PET $(97.0 \%)$ was higher than that of CT $(87.9 \%)$, while the accuracy of detection of nodal involvement was $67.7 \%$ by PET and $75.8 \%$ by CT.
Table 4. Detection of nodal involvement by FDG-PET and CT according to location of involved lymph nodes

\begin{tabular}{lclc}
\hline & & \multicolumn{2}{c}{$\begin{array}{c}\text { Detection of involved } \\
\text { lymph node }\end{array}$} \\
\cline { 3 - 4 } Metastasis & No. of patients & FDG-PET & CT \\
\hline pN0 & 33 & $1(3 \%)$ & $4(12.1 \%)$ \\
pN1 & 14 & $3(21.4 \%)$ & $8(57.1 \%)$ \\
pN2 & 13 & $6(46.2 \%)$ & $8(61.5 \%)$ \\
pN3 & 2 & $1(50.0 \%)$ & $2(100 \%)$ \\
Total & 62 & $11(17.7 \%)$ & $18(29.0 \%)$ \\
\hline
\end{tabular}

Sensitivity: FDG-PET, 34.5\%; CT, 62.1\%; specificity: FDG-PET, 97.0\%; CT, 87.9\%; accuracy: FDG-PET, 67.7\%; CT, 75.8\%

\section{Preoperative detection of other tumors and distant metastasis}

Preoperative FDG-PET detected colon cancer in two patients, adrenal tumor in one patient, lung cancer in one patient and lung metastasis in one patient.

\section{Discussion}

The usefulness of FDG-PET for diagnosis has been established in various tumors, such as head and neck tumors [8], lung cancer [9], colon cancer [10], and esophageal cancer [11]. However, the usefulness of FDG-PET for the detection of gastric cancer has remained unclear. In this study, we evaluated the usefulness of preoperative FDG-PET for the detection of gastric cancer according to histological type, depth of invasion, and nodal involvement, and for the detection of other malignancies.

\section{Sensitivity of detection of primary gastric cancer} by FDG-PET

The sensitivity of the detection of primary gastric cancer by FDG-PET has been reported to be $60 \%-94 \%$ [2-4]. In these previous reports however, many subjects had advanced cancers, and none or few had EGCs. In the present study, the sensitivity of detection of the primary tumor by FDG-PET in the patients overall was low at $58 \%$. However, for those with advanced gastric cancer it was $83 \%$, which was comparable with that in previous reports.

In contrast to colon cancer, gastric cancer exhibits various histological types. Stahl et al. [2] examined the sensitivity of FDG-PET for detecting locally advanced gastric cancer. They reported that the detection rate of the diffuse type, according to Lauren's classification, (41\%) was significantly lower than that of the intestinal type $(83 \%)$, and they noted that this phenomenon may be due to the mucin content in diffuse-type gastric 
cancer, which has a diffuse growth pattern. Their data are supported by the findings of another study, showing low expression of glucose transporter-1 (Glut-1) in signet ring cells and mucinous gastric carcinoma [12].

In the present study, the detection rate of the intestinal type (65.5\%) was higher than that of the diffuse type $(51.5 \%)$ overall, but the difference was not significant. Mochiki et al. [3] also reported no difference in the detection rates of the intestinal type (72\%) and the diffuse type (78\%). However, among the EGCs in the present study, the detection rate of the intestinal type $(43.8 \%)$ was significantly higher than that of the diffuse type $(0 \%)$.

There have been few reports about the sensitivity of EGC detection by PET. Mochiki et al. [3] reported that the detection rate of EGC in their study was $40 \%(10 /$ $25)$. In our study, the detection rate of EGC was $25.9 \%$, and all detected EGCs were of the intestinal type, as seen in Table 2.

It is noteworthy that two of the seven PET-positive patients with EGC had nodal involvement. The uptake of FDG in malignant cells is reported to be correlated with the tumor growth rate [13] and with the number of viable cells [14]. Also, Kawamura et al. [12] found that the expression of glucose transporter-1 (Glut-1) in gastric cancer was associated with parameters of aggressiveness of the tumor, such as depth of invasion, lymphatic permeation, venous invasion, nodal involvement, and hepatic metastasis. Therefore, a positive PET finding may demonstrate malignant aggressiveness, even in EGCs, and this may be an indicator of the extent of the lymphadenectomy required.

The sensitivity of detection of nodal involvement and the accuracy shown by PET were lower than those shown by CT. However, the specificity of detection of nodal involvement by PET was higher than that of CT. These data are consistent with those of previous reports $[3,4]$. However, images obtained by the fusion of PET and CT enable the detection not only of morphologic changes such as the size or shape of the involved lymph node but also of the metabolic abnormalities of the involved lymph node. Chen et al. [4] reported that FDG-PET upstaged $6 \%$ of patients from false-negative CT findings and downstaged $9 \%$ of patients from falsepositive CT findings, and that the patients who benefited from the FDG-PET detection method were given a timely curative resection, without the need for any extra neoadjuvant chemotherapy. Thus, to detect involved lymph nodes, fusion images of PET and CT may be more useful and informative than CT alone.

The detection of nodal involvement by PET according to the location of involved lymph nodes, according to the guidelines of the Japanese Gastric Cancer Association, may help to ensure adequate lymphadenectomy. Mochiki et al. [3] reported that FDG-PET appeared to provide important additional information concerning the aggressiveness of the tumor and prognosis in patients with gastric cancer.

Finally, we found that preoperative PET was useful, in five patients, for the detection of other malignancies or distant metastasis. Such results add to the advantages of using FDG-PET preoperatively in gastric cancer patients.

In conclusion, larger or more advanced tumors with nodal involvement had a higher detection rate by PET. In EGCs, only the intestinal type was detectable by PET. PET-positive EGCs may be aggressive and carry a high possibility of nodal involvement. Preoperative PET also detected other malignancies and distant metastasis.

Although preoperative PET is not essential for all gastric cancer patients, we recommend that it be performed preoperatively, for the following reasons:

(1) Intestinal-type EGCs that are PET-positive have a high possibility of regional lymph node involvement. Adequate lymph node dissection is needed for patients with such results.

(2) In patients with AGC, because almost all PETpositive lymph nodes proved to be involved, we would be able to detect the sites of involved lymph nodes accurately, by using PET combined with CT. Thus, we would be able to plan adequate lymph node dissection preoperatively.

(3) Not only distant metastases but also other cancers can be found by preoperative PET.

\section{References}

1. Yeung HW, Macapinlac H, Karpeh M, Finn RD, Larson SM. Accuracy of FDG-PET in gastric cancer. Preliminary experience. Clin Positron Imaging 1998;1:213-21.

2. Stahl A, Ott K, Weber WA, Becker K, Link T, Siewert JR, et al. FDG PET imaging of locally advanced gastric carcinomas: correlation with endoscopic and histopathological findings. Eur J Nucl Med 2003;30:288-95.

3. Mochiki E, Kuwano H, Katoh H, Asao T, Oriuchi N, Endo K. Evaluation of 18F-2-deoxy-2-fluoro-D-glucose emission tomography for gastric cancer. World J Surg 2004;28:247-53.

4. Chen J, Cheong JH, Yun MJ, Kim J, Lim JS, Hyung WJ, et al. Improvement in preoperative staging of gastric adenocarcinoma with positron emission tomography. Cancer 2005;103:2383-90.

5. Koga H, Sasaki M, Kuwabara Y, Hirata K, Nakagawa M, Abe K, et al. An analysis of the physiological FDG uptake pattern in the stomach. Ann Nucl Med 2003;17:733-8.

6. Japanese Gastric Cancer Association. Japanese classification of gastric carcinoma: 2nd English edition. Gastric Cancer 1998;1:1024.

7. Hamilton SR, Aaltonen LA. Tumors of the stomach. In: Hamilton S, Aaltonen L, editors. WHO classification of tumors. Pathology and genetics, tumors of the digestive system. Lyon: IARC; 2000. p. 38-52.

8. Stokkel MP, Broek FW, Hordijk GJ, Koole R, van Rijk PP. Preoperative evaluation of patients with primary head and neck cancer using dual-head 18-fluorodeoxyglucose positron emission tomography. Ann Surg 2000;231:229-34. 
9. Pieterman RM, van Putten JW, Meuzelaar JJ, Mooyaart EL, Vaalburg W, Koëter GH, et al. Preoperative staging of nonsmall-cell lung cancer with positron-emission tomography. N Engl J Med 2000;254-61.

10. Flamen P. Positron emission tomography in colorectal cancer. Best Pract Res Clin Gastroenterol 2002;16:237-51.

11. Flamen P, Lerut A, Van Cutsem E, De Wever W, Peeters M, Stroobants S, et al. Utility of positron emission tomography for the staging of patients with potentially operable esophageal carcinoma. J Clin Oncol. 2000;18:3202-10.

12. Kawamura T, Kusakabe T, Sugino T, Watanabe K, Fukuda T, Nashimoto A, et al. Expression of glucose transporter-1 in human gastric carcinoma. Cancer 2001;92:634-41.
13. Ohira H, Kubota K, Ohuchi N, Harada Y, Fukuda H, Satomi S. Comparison of intratumoral distribution of ${ }^{99 \mathrm{~m} T c-M I B I}$ and deoxyglucose in mouse breast cancer models. J Nucl Med 2000;41: 1561-8.

14. Kubota K, Ishiwata K, Kubota R, Yamada S, Tada M, Sato T, et al. Tracer feasibility of monitoring tumor radiotherapy: a quadruple tracer study with fluorine-18-fluorodeoxyglucose or fluorine-18-fluorodeoxyuridine, L-[methyl $\left.-{ }^{14} \mathrm{C}\right]$ methionine, $\left[6-{ }^{3} \mathrm{H}\right]$ thymidine, and gallium-67. J Nucl Med 1991;2118-23. 